\title{
INTERPOLATION-BASED RECONSTRUCTION METHODS FOR TOMOGRAPHIC IMAGING IN 3D POSITRON EMISSION TOMOGRAPHY
}

\author{
Yingbo LI*, ANton KUMMERT *, Fritz BOSCHEN *, HANS HERZOG ** \\ * Faculty of Electrical, Information and Media Engineering \\ University of Wuppertal, Rainer-Gruenter-Street 21 \\ 42119 Wuppertal, Germany \\ e-mail: \{yingbo, kummert, boschen@uni-wuppertal.de\} \\ ${ }^{* *}$ Institute of Medicine, Research Centre Juelich \\ 52428 Juelich, Germany \\ e-mail: h.herzog@fz-juelich.de
}

\begin{abstract}
Positron Emission Tomography (PET) is considered a key diagnostic tool in neuroscience, by means of which valuable insight into the metabolism function in vivo may be gained. Due to the underlying physical nature of PET, 3D imaging techniques in terms of a 3D measuring mode are intrinsically demanded to assure satisfying resolutions of the reconstructed images. However, incorporating additional cross-plane measurements, which are specific for the 3D measuring mode, usually imposes an excessive amount of projection data and significantly complicates the reconstruction procedure. For this reason, interpolation-based reconstruction methods deserve a thorough investigation, whose crucial parts are the interpolating processes in the 3D frequency domain. The benefit of such approaches is apparently short reconstruction duration, which can, however, only be achieved at the expense of accepting the inaccuracies associated with the interpolating process. In the present paper, two distinct approaches to the realization of the interpolating procedure are proposed and analyzed. The first one refers to a direct approach based on linear averaging (inverse distance weighting), and the second one refers to an indirect approach based on two-dimensional convolution (gridding method). In particular, attention is paid to two aspects of the gridding method. The first aspect is the choice of the two-dimensional convolution function applied, and the second one is the correct discretization of the underlying continuous convolution. In this respect, the geometrical structure named the Voronoi diagram and its computational construction are considered. At the end, results of performed simulation studies are presented and discussed.
\end{abstract}

Keywords: Tomographic reconstruction, three-dimensional positron emission tomography, Fourier slice theorem, frequency sample distribution, two-dimensional interpolation, inverse distance weighting, gridding method.

\section{Introduction}

With the aid of Computerized Tomographic (CT) imaging methods, the human ability to gain non-invasive insight into the internal structure of living organisms can be significantly extended. Among the broad spectrum of various imaging techniques, Positron Emission Tomography (PET) is regarded as a key diagnostic tool in neuroscience for studying the metabolism function in vivo (Beutel et al., 2000). During the measuring procedure of PET, tracer substances containing neutron-deficient radioisotopes have to be injected into the human body at first, to spread in accordance with the metabolism in the body and reach a quasi-steady distribution in the organ of interest afterwards. Since the half-life period of utilized radioisotopes is relatively short, the nuclear decay of the neutron-deficient nuclide in terms of emitting positrons may occur shortly after the injection. However, each of the emitted positrons collides nearly immediately with an available electron in tissues so that the so-called annihilation process takes place. The two annihilation photons, which emerge during this process, have very high departing velocities and may leave the human body in all likelihood along opposed directions. In this respect, if two distinct photons are detected by two different sensor units of the measuring system within a predefined time win- 
dow, this pair of photons can be assumed to belong to the same annihilation process and can be hence registered as a single coincidence event. Its true occurring location, where the positron-emitting radioisotope actually locates, lies somewhere on the straight line between these two detecting sensor units. Thereafter, in the absence of effects such as attenuation and scattering, the measured number of coincidence events along the individual straight Line-Of-Response (LOR) between two sensor units approximates the straight line integral of the underlying tracer substance distribution. Since such substance distributions normally reflect the metabolic functions in vivo, malfunctions which are characteristic for diverse functional disorders could be diagnosed by observing the proper visualization of the non-invasive distribution.

Due to the physical nature of the annihilation process, the two emerging photons are actually emitted into all spatial directions, i.e., there is no preferential direction in the three-dimensional (3D) spatial domain. Correspondingly, 3D imaging techniques are intrinsically demanded for PET, since the more coincidence events are measured by the measuring system of the PET scanner, the better reconstruction quality may be achieved. To fulfill this requirement, the measuring systems of most PET scanners nowadays have cylindrical multi-ring structures whose cylindrical surfaces are subdivided into uniform sensor block units in both transaxial and axial directions (Bendriem and Townsend, 1998). For convenience, the axial direction is conventionally designated as the $z$ direction, whereas the transverse ring plane corresponds to the $x$-y-plane, see Fig. 1. In order to enhance the system sensitivity, not only the sensor units on the same rings but also the ones on the different rings can be interconnected electrically. Thereby the originated large axial field of view permits measurements of the so-called crossplane coincidence events and enables the achievement of better reconstruction quality. To distinguish it from from the obsolete two-dimensional (2D) measuring mode, such an imaging technique in PET is henceforth designated as 3D-PET.

On the other hand, the incorporation of additional cross-plane events often results in an excessive amount of projection data, which significantly complicates the reconstruction procedure. By using standardized reconstruction algorithms such as analytical Filtered-BackProjection (FBP) or the iterative MaximumLikelihood-Expectation-Maximization (MLEM) method, clinically impractical reconstruction time durations become necessary (Moon, 1996). For this reason, reconstruction approaches based on the Fourier slice theorem, by means of which spectral values at particular frequency sampling points in the discrete object spectrum can be obtained, deserve a thorough investigation. Starting from those frequency samples, the original distribution function in the spatial domain can be directly calculated by perfor- ming the inverse Fourier transform. Because of the simplicity of such approaches, the reconstruction time can be considerably reduced. Since the available sampling points in the 3D frequency domain are naturally unevenly distributed, theoretically the time-consuming Discrete Fourier Transform (DFT) should be applied to perform the inverse transform. In practice, an additional interpolating step for estimating the unknown spectral values on a predefined Cartesian grid is usually inserted to enable the utilization of the Fast Fourier Transform (FFT) algorithm. Once the interpolation is accomplished, the sought-after discrete distribution function can be calculated by performing the inverse FFT. In this way, the reconstruction can be sped up thanks to the FFT's speed advantage. Due to the usage of the interpolating step, such reconstruction approaches are referred to as interpolation-based reconstruction methods in this paper and will be closely addressed.

In the present paper, the principle of interpolationbased reconstruction approaches will be presented first. Apparently, their crucial parts are the interpolating steps in the frequency domain which are always associated with inevitable inaccuracies (Thevenaz et al., 2000). In the 2D measuring mode, the fact that the sampling points with known spectral values are highly unevenly distributed leads to a very tricky problem. In the low frequency regions, the sample densities are still high enough to guarantee the interpolation results, but in the high frequency regions the sample densities are simply too low to ensure achieving rational results. Compared with the $2 \mathrm{D}$ measuring mode, this problem still remains in 3D-PET but turns out to be less critical because there is a larger number of frequency samples available in the frequency domain due to the additional cross-plane events. This is particularly beneficial for minimizing the unavoidable artifacts introduced by the interpolation procedure. Unfortunately, some analysis reveals also the side-effect of this benefit ( $\mathrm{Li}$ et $a l ., 2005)$. The fluctuation of the sample density becomes also much more unpredictable, which increases the complexity of a rational implementation. Based on this fact, two distinct realizations of the interpolation procedure are pursued. The first approach uses the straightforward linear averaging method to estimate the unknown spectral values, whereas the second approach applies convolution operations to obtain the values at desired interpolation points. Simulation results of these two approaches are presented subsequently. Due to the achieved different performances, specific details and possible reasons are presented and discussed at the end.

\section{Principle of the interpolation-based reconstruction approach}

The fundamental concept of interpolation-based reconstruction methods in 3D-PET is given by the 3D Radon transform, which relates the measurable projection data to 


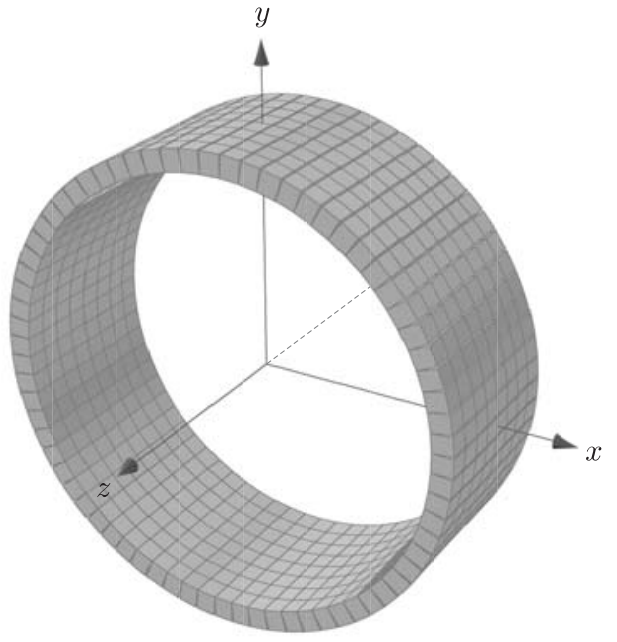

Fig. 1. Schematic illustration of the sensor arrangement of a PET scanner.

the underlying continuous tracer distribution via integrals along straight lines (Kak and Slaney, 1988). With the 3D tracer distribution defined as $f(x, y, z)$, the mathematical definition of the associated projection signal $p(u, v, \vartheta, \varphi)$ at a certain projection angle $(\vartheta, \varphi)$ can be formulated as

$$
p(u, v, \vartheta, \varphi)=\int_{-\infty}^{\infty} f(x, y, z) \mathrm{d} t
$$

where the involved 3D coordinate transform is defined by

$$
\begin{aligned}
{\left[\begin{array}{l}
x \\
y \\
z
\end{array}\right]=} & {\left[\begin{array}{ccc}
\cos \varphi \cos \vartheta & -\sin \varphi & -\cos \varphi \sin \vartheta \\
\sin \varphi \cos \vartheta & \cos \varphi & -\sin \varphi \sin \vartheta \\
\sin \vartheta & 0 & \cos \vartheta
\end{array}\right] } \\
& \cdot\left[\begin{array}{l}
t \\
u \\
v
\end{array}\right] .
\end{aligned}
$$

The spatial slope of an individual straight line is determined by the azimuthal angle $\varphi$ and the co-polar angle $\vartheta$ with respect to the transverse ring plane, while the parameters $u$ and $v$ depict its displacement with respect to the origin in the space domain.

In accordance with the definition (1), the originally 2D Fourier slice theorem may be generalized to

$$
\begin{aligned}
& P\left(j \omega_{u}, j \omega_{v}, \vartheta, \varphi\right) \\
& =F\left(j \omega_{x}, j \omega_{y}, j \omega_{z}\right) \mid \begin{array}{l}
\omega_{x}=-\omega_{u} \sin \varphi-\omega_{v} \cos \varphi \sin \vartheta \\
\omega_{y}=\omega_{u} \cos \varphi-\omega_{v} \sin \varphi \sin \vartheta \\
\omega_{z}=\omega_{v} \cos \vartheta
\end{array}
\end{aligned}
$$

which implies that the 2D Fourier transform of the parallel projections $p(u, v, \vartheta, \varphi)$ with respect to the varia- bles $u$ and $v$ at a certain projection angle $(\vartheta, \varphi)$ corresponds to a central plane crossing the 3D Fourier transform of $f(x, y, z)$ at exactly the same projection angles, see Fig. 2. Theoretically, with the help of the extended 3D Fourier Slice Theorem in (2), the 3D object spectrum $F\left(j \omega_{x}, j \omega_{y}, j \omega_{z}\right)$ could be entirely recovered by acquiring 2D projection spectra at all feasible projection angles. Despite possible data redundancy, the desired tracer distribution could then be figured out by performing the inverse Fourier transform (Li et al., 2005). Unfortunately, this procedure is merely applicable for the continuous case. In practice, the number of available projection angles as well as the number of projections at each projection angle are strictly limited. In this note, the reconstructed 3D object spectrum is no longer of continuous nature, but it is merely composed of frequency samples. Due to the additional cross-plane coincidence events in the $3 \mathrm{D}$ measuring mode, the number of obtainable frequency samples in the frequency domain is larger than that in the $2 \mathrm{D}$ measuring mode. For an interpolation-based reconstruction approach, the exact arrangement of these frequency samples in the 3D frequency domain has to be ascertained for the subsequent interpolating procedure.

\section{Distribution of frequency samples in the 3D frequency domain}

With the measurable projection data defined as straight line-integrals, projections having the same inclination can be combined together as sets of parallel line-integrals. Although the sensor system of a modern PET scanner has actually a cylindrical multi-ring structure, the measured coincidence events can be subsequently arc-corrected and rearranged in such a way as if they were consecutively captured by two simultaneously rotating, parallel sensor panels at various projection angles, see Fig. 3. It is worth emphasizing here that despite the slope of the two projection panels with the angle $\vartheta$ in the axial direction, the rota-

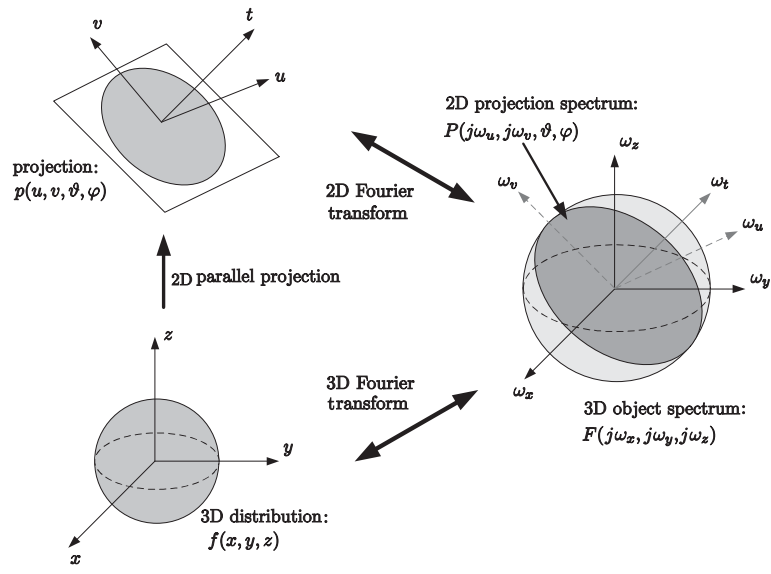

Fig. 2. Schematic illustration of the 3D Fourier slice theorem. 
tional axis of the rotational movement remains the $z$-axis. The individual projection beam which is determined by the two associated block-detectors is conventionally designated as the Tube Of Response (TOR). Along with this modeling scheme, a related fictitious projection plane can be introduced at each projection angle, which is parallel to the two-sensor panels and additionally passes through the origin of the spatial domain. If the inclination of a projection plane is defined as the direction of its normal, the inclination of a projection plane is then consistent with the associated projection angle.

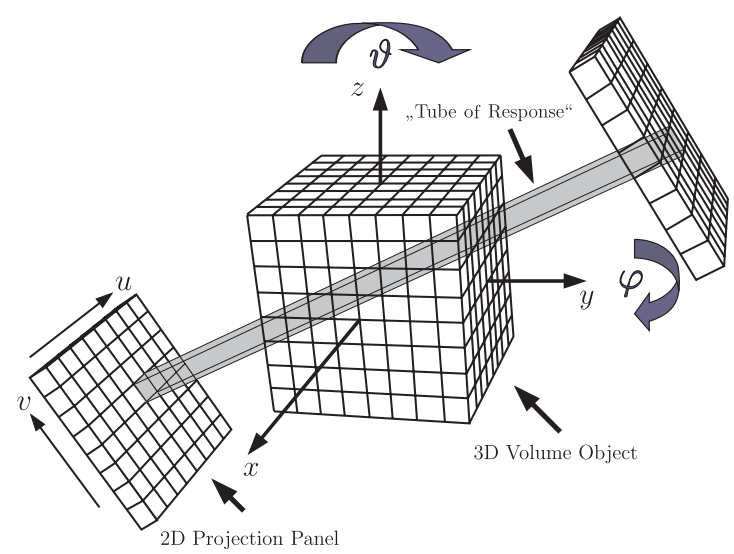

Fig. 3. Modeling scheme of a cylindrical sensor system as two simultaneously rotating sensor panels.

According to this definition, in the case of the 2D measuring mode the projection plane has definitely no inclination with respect to the $z$-axis, whereas in the case of the 3D measuring mode the projection plane correlating with cross-plane coincidence events may be inclined with respect to the $z$-axis. In the latter case, the associated co-polar angle $\vartheta$ has a nonzero value. Due to the geometrical modeling scheme of the PET scanner, the measurable parallel projection samples in a projection plane are intrinsically equidistantly arranged along the $z$-axis. On the contrary, along the perpendicular transverse direction an arc-correction step has to be performed to ensure the equidistance between adjacent projection samples, which is normally already integrated into the projection acquisition procedure of hardware and hence causes no additional computational overhead.

To this end, the projection samples in an arbitrary projection plane are indeed ordered on a Cartesian gird. Thereupon, the 2D fast Fourier transform can be utilized to compute the discrete projection spectrum economically, while the frequency samples are arranged on a Cartesian grid as well. Considering the multi-ring block-detector structure of a PET scanner, the spatial distribution of frequency samples in the reconstructed 3D object spectrum can be indeed constituted by consecutively gathering the frequency samples with distinct inclinations, as if the 2D
Cartesian lattices of frequency samples were revolved in the 3D frequency domain. In Fig. 4 such a constitution procedure is schematically illustrated. For the 2D measuring mode, the revolving Cartesian lattice in the frequency domain has no slope with respect to the $\omega_{z}$-axis so that the resulted frequency samples are bounded within a cylindrical scope, see Fig. 4. In the 3D measuring mode, however, apart from the projections along the transverse direction, the revolving 2D Cartesian lattices correlating with cross-plane events definitely tilt to the $\omega_{z}$-axis. In this case, only part of the object spectrum can be reconstructed. The confined region in the 3D frequency domain, in which no frequency samples are obtainable, resembles a head-to-head adhered truncated double-cone, see Fig. 4 The final distribution of frequency samples in the reconstructed 3D object spectrum is hence the accumulation of all acquirable frequency samples, no matter whether from the inclined or the noninclined Cartesian lattices.

The resulting frequency samples are obviously irregularly distributed in the frequency domain. For the sake of rapid reconstruction, interpolating steps have to be conducted to enable the usability of the FFT algorithm. Theoretically, a true $3 \mathrm{D}$ interpolating procedure in the $3 \mathrm{D}$ frequency domain seems to be inevitable. But by observing it closely, it turns out that only consecutive 2D interpolations in a set of transverse planes are really required, because the obtainable frequency samples fall exactly in these equidistantly displaced planes which are perpendicular to the $\omega_{z}$-axis (Li et al., 2006). This characteristic simplifies the computational complexity of the interpolating procedure significantly and speeds up the reconstruction again. Generally speaking, incorporating cross-plane measurements leads to an increased density of frequency samples in all transverse planes, which induces to scale down the interpolation error effectively. However, depending on the axial location of the transverse plane, the density increase fluctuates considerably in various regions of

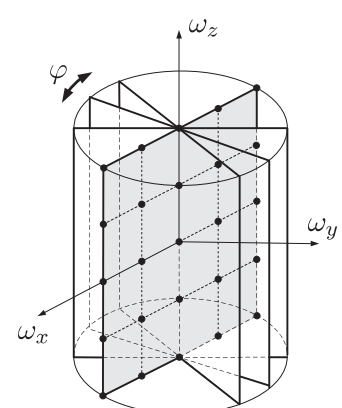

(a)

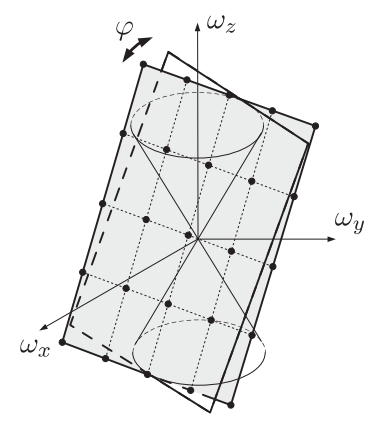

(b)
Fig. 4. Schematic illustration of the frequency sample structure in the reconstructed 3D object spectrum: (a) Fourier transformed projection plane with no inclination , (b) Fourier transformed projection plane with inclination. 
transverse planes. For a transverse plane lying closely to the origin of the 3D frequency domain, the additional frequency samples concentrate predominantly in the central region, where the density is already high enough for rational interpolation, see Fig. 5 Otherwise, for a transverse plane lying comparatively far from the spectral origin, the predominant concentration of additional frequency samples shifts outwards, see Fig. 5. From the experience gained during the simulation, the density increase of frequency samples in the border area is actually more beneficial for improving the interpolation results than the density increase in the central region of an individual transverse plane, because the density of only obtainable frequency samples in the 2D measuring mode is too low for a rational interpolation in the border region.

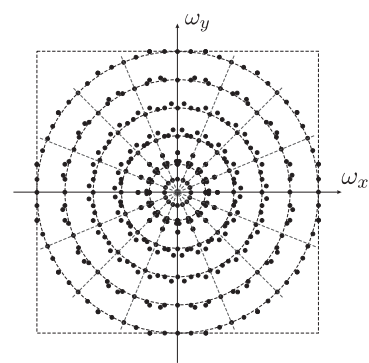

(a)

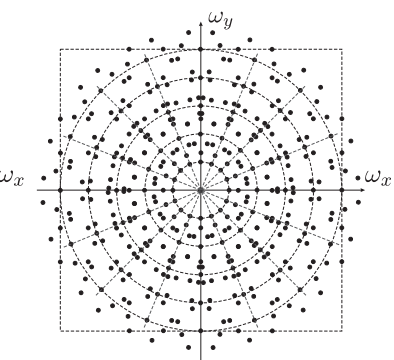

(b)
Fig. 5. Schematic illustration of frequency sample distributions in distinct transverse planes in the reconstructed 3D object spectrum: (a) distribution of frequency samples in a transverse plane lying close to the spectral origin, (b) distribution of frequency samples in a transverse plane lying far from the spectral origin.

\section{Consecutive 2D interpolation procedures in the 3D frequency domain}

For the purpose of speeding up the reconstruction procedure, interpolation operations have to be performed in each transverse plane of the reconstructed 3D object spectrum to enable the usability of the FFT algorithm. This procedure is illustrated schematically in Fig. 6. Although the intrinsic error associated with the interpolating procedure cannot be completely avoided, distinct interpolating schemes do provide varying performances. In the present paper, two distinct interpolating approaches are pursued and analyzed. The first approach presents a straightforward solution, for which the unknown spectral values are estimated by the weighted linear averaging of the spectral values of the relevant neighboring frequency samples. On the contrary, the second approach uses the 2D convolution operations in each transverse plane to acquire the unknown values at the interpolation sites. Due to the 2D convolution operation in the frequency domain, an addi-

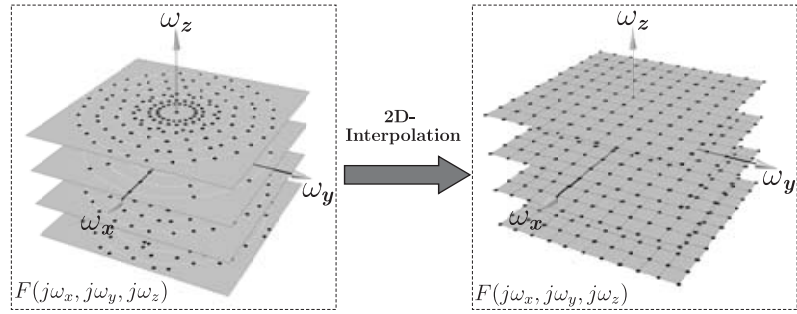

Fig. 6. Illustration of the interpolating procedure in all transverse planes.

tional division step in the spatial domain has to be accomplished subsequently to acquire the correct results.

4.1. Inverse distance weighting interpolation. The Inverse Distance Weighting (IDW) interpolation method, which was originally utilized in geoinformatics, can be easily adopted for the case of interpolating from 2D unevenly distributed frequency samples onto frequency samples arranged on a Cartesian grid in the transverse plane. The IDW method is based on the assumption that the frequency sample which is to be interpolated should be more influenced by the closely located than by the remotely located neighboring samples (Fisher and Embleton, 1987). In this respect, the value at the desired location is then a weighted linear average of the neighboring values, whereas the associated weight decreases if the distance between the interpolation site and the scattered neighbor increases. The corresponding mathematical definition of IDW interpolation can be therefore formulated as

$$
F_{\text {regular }}=\sum_{i=1}^{N} w_{i} \cdot f_{i}, \quad \text { with } w_{i}=\frac{h_{i}^{-p}}{\sum_{j=1}^{N} h_{j}^{-p}},
$$

where $f_{i}$ represents the spectral value of the $i$-th scattered frequency sample and $w_{i}$ the associated weight. In the formula for calculating individual $w_{i}, h_{j}$ depicts the distance between the $j$-th neighboring sample and the interpolation site, while $N$ is the total number of all existing neighbors within a pre-specified neighborhood. In Fig. 77 the selection of a relevant neighborhood and the related neighboring samples are exemplified, where the pre-defined relevant neighborhood is illustrated as a gray-shaded square.

In fact, the larger the relevant neighborhood is selected, the larger the number of scattered frequency samples may be incorporated into the interpolating procedure, and in turn the more accurate interpolation results could be expected. However, a large number of sample neighbors means also a higher computational expense, which correspondingly slows down the reconstruction speed. Therefore, a trade-off between the reconstruction time and the interpolation accuracy has to be made. In the pre- 
sent paper, two different solutions are proposed in this respect. On the one hand, the neighborhood's extent is uniformly defined independently of the related frequency sample densities. The drawback of this easily realizable approach is that the number of incorporated frequency samples is permanently variable. Particularly in the border region of the traverse plane, the number of samples could be very low because neighboring samples for a specified interpolation site are often barely available. On the other hand, instead of a uniform extent of neighborhood an equal number of neighboring samples can be used as the criterion to determine the unequally bounded neighborhood. In the border region with a low sample density, the seeking area has to be expanded gradually until enough neighboring samples are encountered. Compared with the former case, this seeking procedure is much more time consuming. Fortunately, the exact locations of unevenly distributed frequency samples in each transverse plane can be determined in advance, because they are only affected by the geometry of the PET scanner. Consequently, the unevenly distributed frequency samples in each plane can be sorted according to their locations by using an efficient data structure such as the balanced binary tree. Based on the specified neighboring samples, the associated weights $w_{i}$ can be directly calculated according to (3). Since the locations of the frequency samples as well as their associated weights can be calculated without knowledge about their spectral values, the seeking and the successive calculating procedure can be completed prior to the actual reconstruction. In this way, the reconstruction duration can be kept short.

4.2. Gridding method. The second approach to estimate the spectral values on a specified 3D Cartesian lattice is based on separate $2 \mathrm{D}$ convolution operations in each transverse plane. This approach was conventionally used in the $2 \mathrm{D}$ reconstruction scenario and embraced by the term "gridding method" (Schomberg and Timmer, 1995). Since the irregularly distributed frequency

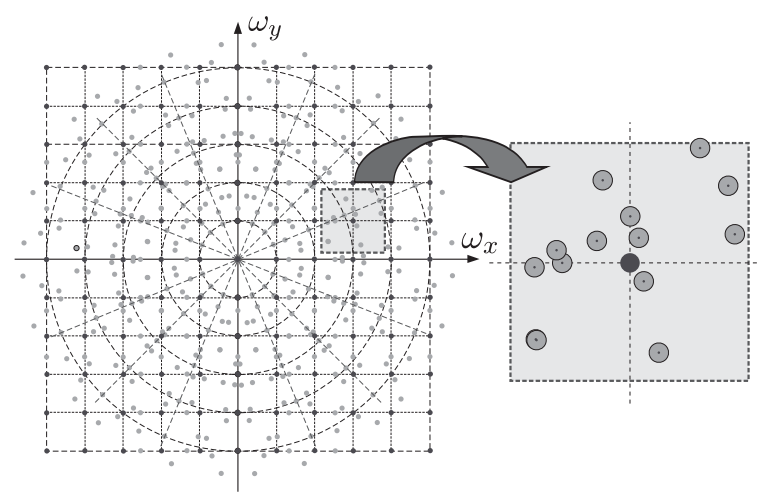

Fig. 7. Selection of the relevant neighborhood for a frequency node in the transverse plane to be interpolated. samples in the 3D frequency domain merely locate in certain transverse planes, the gridding method known from the $2 \mathrm{D}$ scenario can be easily extended to the $3 \mathrm{D}$ reconstruction scenario, whose functional principle is outlined in the following way: Consider a discrete 3D object spectrum $F_{\text {irregular}}$, whose frequency samples are irregularly distributed in a set of equidistantly displaced transverse planes in the frequency domain. The equivalent discrete spectrum $F_{\text {Cartesian }}$, but with sampling points arranged on a 3D Cartesian grid, can be obtained by consecutively performing 2D convolutions with a predefined 2D window function $W_{\text {window }}$ in all the transverse planes:

$$
\left\{F_{\text {Cartesian }}\right\}_{\text {layer }}=\left\{F_{\text {irregular }}\right\}_{\text {layer }} * W_{\text {window }},
$$

where $*$ denotes the $2 \mathrm{D}$ convolution operation and the subscript $\{\cdot\}_{\text {layer indicates that the convolution operation }}$ merely takes place layer-wise. Due to the consecutive 2D convolutions, the obtained $F_{\text {Cartesian }}$ actually is not the sampled object spectrum of the desired distribution function $f_{\text {Cartesian }}$, but rather an intermediate outcome. In order to compensate the effect of convolution in the frequency domain, an additional division step in the spatial domain has to be introduced in the inversion step, prior to a straightforward 3D inverse Fourier transform. With $\mathcal{F}_{x y}^{-1}$ defined as the 2D inverse Fourier transform in each transverse plane with respect to the variables $x$ and $y$, and $\mathcal{F}_{z}^{-1}$ defined as the 1D inverse Fourier transform with respect to the variable $z$, respectively, the correct inversion procedure to acquire the desired $f_{\text {Cartesian }}$ can be ultimately expressed by the formula

$$
f_{\text {Cartesian }}=\mathcal{F}_{z}^{-1}\left\{\frac{\mathcal{F}_{x y}^{-1}\left\{F_{\text {Cartesian }}\right\}_{\text {layer }}}{w_{\text {window }}}\right\},
$$

where $w_{\text {window }}$ denotes the $2 \mathrm{D}$ inverse Fourier transform of the $2 \mathrm{D}$ convolution window $W_{\text {window }}$ used in the frequency domain.

Despite the seemingly uncomplicated expression in (5), two significant aspects of the gridding method have to be carefully considered. The first one is the selection of the 2D window function $W_{\text {window }}$. Theoretically, the optimal convolution function in the frequency domain should be of infinite extent, which is, however, impractical with respect to the computational cost. For this reason, the convolution function has to be truncated and windowed, so that a trade-off between the reduced computational effort and the accompanied accuracy deficit has to be taken into account. Several authors have been engaged in this aspect for finding an optimally appropriate solution and suggested the Modified Kaiser-Bessel (MKB) window function as the most promising candidate (Schomberg and Timmer, 1995; Jackson et al., 1991; Matej and Lewitt, 2001). Although the conducted works deal mainly with the 2D reconstruction scenario, the proposed MKB window function can be adopted here without significant modification, 
since for 3D-PET merely successive 2D gridding procedures are required. With properly selected parameters, the bell-shaped MKB function and its inverse Fourier transform may be free from discontinuities. Actually, the 2D convolution window used is the multiplicative product of two 1D MKB functions.

The second important aspect of the gridding method is the correct discretization of the underlying $2 \mathrm{D}$ continuous convolution depicted in (4), since in $F_{\text {irregular only }}$ frequency samples are available. In this case, the continuous integral operation must be approximated and replaced by a double summation, where each involved frequency sample receives a weighting factor. Generally speaking, the related weighting factor for a certain frequency sample is inversely proportional to the local density of its neighboring frequency samples. However, since the frequency samples are highly nonuniformly distributed in each transverse plane, accurate calculation of an individual weighting factor poses a computational challenge. In this respect, a new modeling scheme for the assigned weighting factors of scattered frequency samples are proposed, namely, accurately modeled as the normalized area of a polygon, which belongs to the specified frequency sample in the transverse plane. According to this modeling scheme, the higher the local density frequency sample, the smaller the assigned polygon and correspondingly the smaller the related weighting factor. In computational geometry, the subdivision induced by this scheme is called the "Voronoi diagram." Compared with the modeling schemes proposed in the literature so far, the resulting weighting factors are also much more precise in terms of discretization. In contrast to some proposed time-consuming iterative approaches, this modeling scheme is straightforward and time efficient.

4.3. Computational construction of the Voronoi diagram. The Voronoi diagram is a versatile geometric structure. For a set of distinct frequency samples in a transverse plane, the associated Voronoi diagram is defined as the subdivision of the plane into various polygons, one for each sample, with the property that an arbitrary point lies within a specified polygon if and only if the distance from this point to the sample of the associated polygon is shorter than all other distances between this point and the remaining samples (De Berg et al., 1997). Such polygons are often called Voronoi cells, whose normalized area contents are of interest for the gridding method. In Fig. 8, an exemplary Voronoi diagram for some given frequency samples is schematically illustrated. Due to the finite extent of $W_{\text {window }}$, a bounding neighborhood for each interpolation location must be selected first, within which the Voronoi diagram is to be generated.

For computing the Voronoi diagram, Fortune's algorithm commonly known and named after its inventor, is preferred and implemented in this paper (Fortune, 1987).

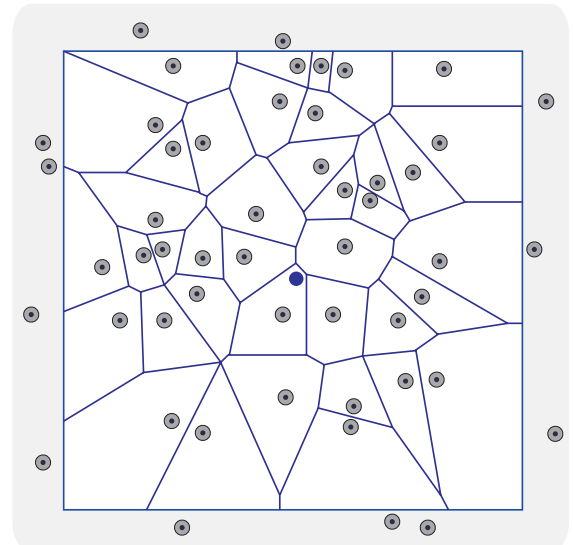

Fig. 8. Schematic illustration of an exemplary Voronoi diagram.

The strategy of this efficient, event-driven algorithm is to sweep a straight line over the concerned transverse plane, during which action two different sorts of events are to be correspondingly handled, the so-called site-event and the arc-event. These two different events can be distinguished subject to the intersection of the already constructed part diagram structure with the sweeping line. In case of encountering a site-event, a new Voronoi cell with associated pending edges has to be added to the existing structure, whereas for encountering an arc-event, the pending edges of an open Voronoi cell are terminated by attaching intersection vertices. The affected Voronoi cell is thus closed. However, after all detected events are handled, there are still some open Voronoi cells. To close them, a bounding box which is equal to the predefined relevant neighborhood has to be added to the existing structure. In this manner the Voronoi diagram, which is only composed of close Voronoi cells, can be constructed step by step till completeness. Once the complete Voronoi diagram is generated, the areal content of each single Voronoi cell can be easily calculated based on its determined vertices and accordingly the weighting factor of the associated frequency sample can be figured out as well.

4.4. Remaining difficulty. Despite this clear construction scheme, there is a remaining difficulty in constructing the Voronoi diagram. Since the encountered events do not have to be handled immediately, they need to be sorted in a queue temporarily subject to their associated coordinates. The coordinate of a site-event is simply the coordinate of the associated frequency sample itself, whereas the coordinate of an arc-event is actually the coordinate of the associated circle's center. Since the associated circle of an arc-event is normally determined by three points during the construction, numerical calculations based on their coordinates are necessary. In this case, numerical inaccuracies cannot be completely excluded. Particularly, since the frequency samples in the transverse plane may be sometimes very densely distributed, numerical inaccuracies 
turn out to be a really serious problem so that in the worst case the affected events may be sorted into a queue with a wrong order. Due to the progressive nature of Fortune's algorithm, even a simply inverted order may lead to a totally false outcome for the Voronoi diagram. Despite great efforts such as iteratively solving the underlying quadratic equation, this problem cannot be optimally and completely avoided so that within the scope of the present paper an additional verifying step has to be accomplished after the constructing procedure. If degeneracy is spotted, the affecting frequency sample has to be regrouped with a nearby neighboring frequency sample and the constructing procedure has to to be repeated under the modified condition. Once the Voronoi diagram is generated, the regrouped frequency samples receive a weighting factor which is equal to the normalized areal content of the Voronoi cell divided by the number of regrouped samples. This means effectively that the erroneously calculated weighting factor has to be accepted to by-pass the numerical problem. However, as the degeneracy occurs seldom and the number of neighboring samples is relatively large, the influence of such approximations could be reasonably neglected.

\section{Simulation results}

For the purpose of evaluation, the two interpolation-based reconstruction methods were implemented using the programming language $\mathrm{C}++$. Although the two proposed methods are generally applicable to truly measured projection data, in the scope of this paper only simulation studies on the basis of simulated projection data are performed. This is due to the fact that the simulated projection data are free from the acquisition noise, which is typical for the truly measured projection data. In the case of using simulated projection data, the reconstruction results are merely influenced by the utilized reconstruction methods and reasonable evaluation with respect to the reconstruction quality may be achieved, whereas in the case of using truly measured projection data, the cause of possible reconstruction deviation cannot be clearly ascertained. For this reason, using noise-free projection data is significant for a correct evaluation of the discussed interpolationbased reconstruction methods.

Consequently, a 3D phantom object consisting layerwise of the so-called 2D Shepp-Logan phantoms is defined as the original distribution function, which is of the dimension $128 \times 128 \times 32$, i.e., 32 layers in the $z$-direction and $128 \times 128$ pixels on each transverse layer. The $2 \mathrm{D}$ Shepp-Logan phantom contains several ellipses with different sizes and absorption properties to resemble the features in the human brain. In order to imitate the 3D brain structure, the extents of the 2D Shepp-Logan phantoms on various layers are intentionally differently specified and the change of extents between layers basically occurs gra-

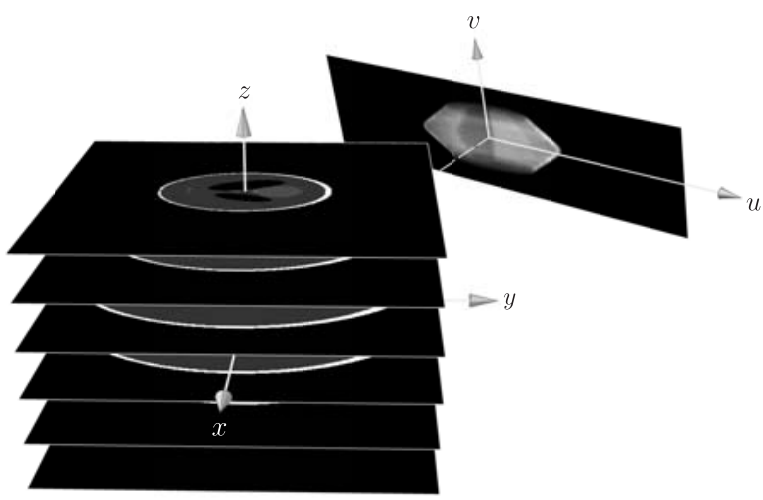

Fig. 9. 3D phantom object consisting layer-wise of 2D Shepp-Logan phantoms.

dually, e.g., the 2D phantoms on the topmost and lowermost layers are much smaller than that on the central layer, see Fig. 9 Based on this defined 3D phantom, the corresponding projection data can be then computed by means of the accurately modeled system matrix, which is primarily based on the geometry of the individual TOR and selfimplemented before (Li et al., 2006).

By using interpolation-based reconstruction methods, i.e., the direct approach (IDW) and the convolutionbased indirect approach (gridding method), distinct reconstruction results of the original 3D phantom can be achieved. In Fig. 10, the layer-wise depiction of the reconstructed 3D phantom object by using the IDW method is given. The observation of this reconstruction result confirms clearly the correctness of the implemented IDW method, in which 2D Shepp-Logan phantoms of various extents in individual transverse layers are properly reconstructed. This conclusion is also applicable to the reconstruction results achieved by using the gridding method. Its complete layer-wise depiction is omitted here, because, due to the restricted depiction extents, differences between the results of the two discussed methods can be barely distinguished optically. Instead, for the purpose of a subjective evaluation, the middle layers of differently reconstructed 3D phantoms as well as the original Shepp-Logan phantom of the same extent are exemplarily selected and depicted side by side in Fig. 11. Although both of the interpolation-based reconstruction methods yield seemingly correct results, optical observation shows anyhow that the reconstruction result achieved by using the IDW method exhibits more distortions than the result achieved by using the gridding method. On the contrary, the difference between the reconstruction results based on the 2D filtered backprojection and the gridding method is minor. Nevertheless, one can say that the contrast in the reconstructed phantom by using the gridding method is slightly higher so that the contour is correspondingly clearer.

As for the simulation time, less than 10 minutes on a $\mathrm{PC}$ are really needed to complete the reconstruction for a 

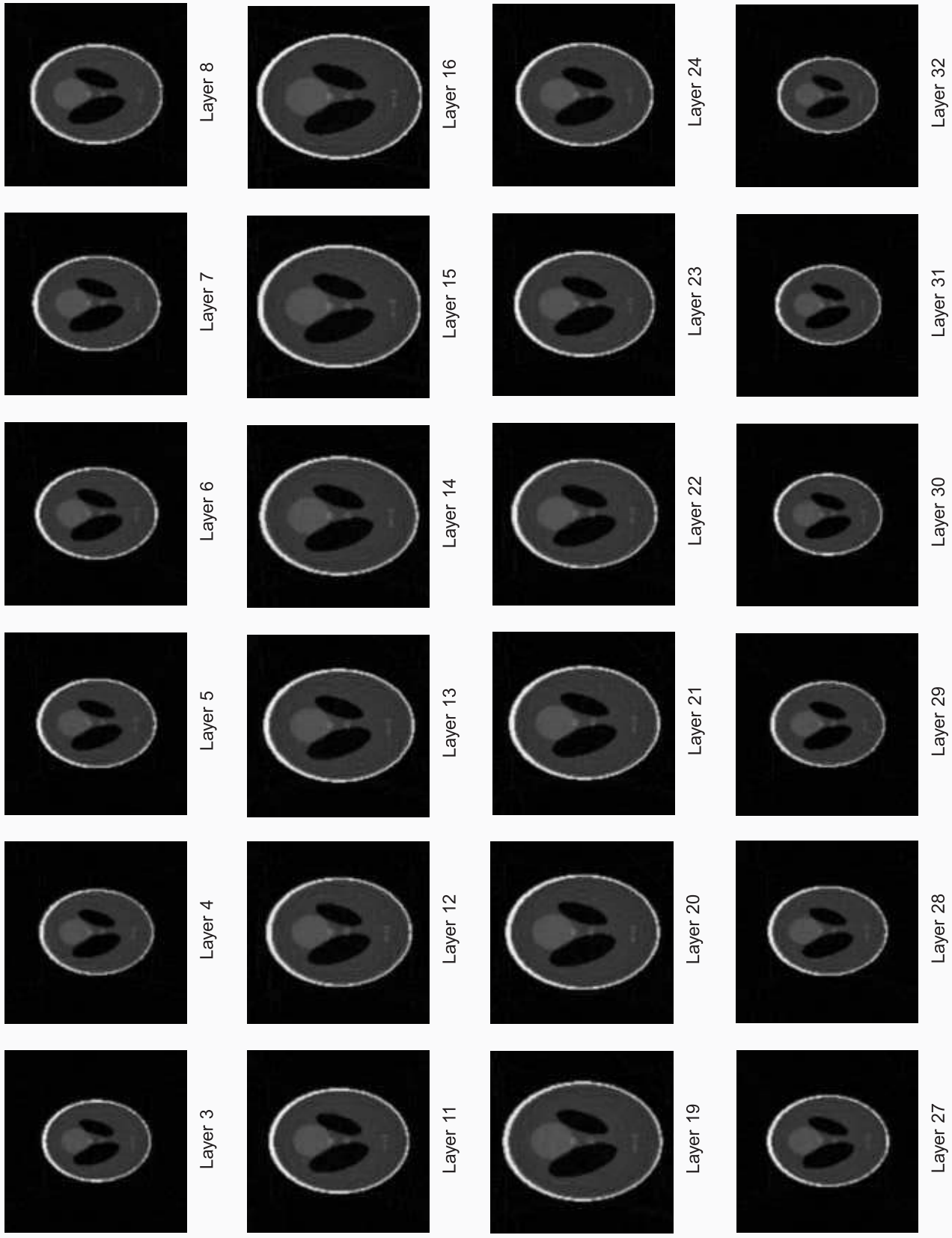

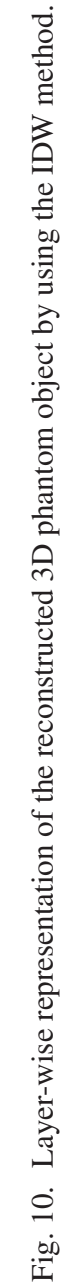
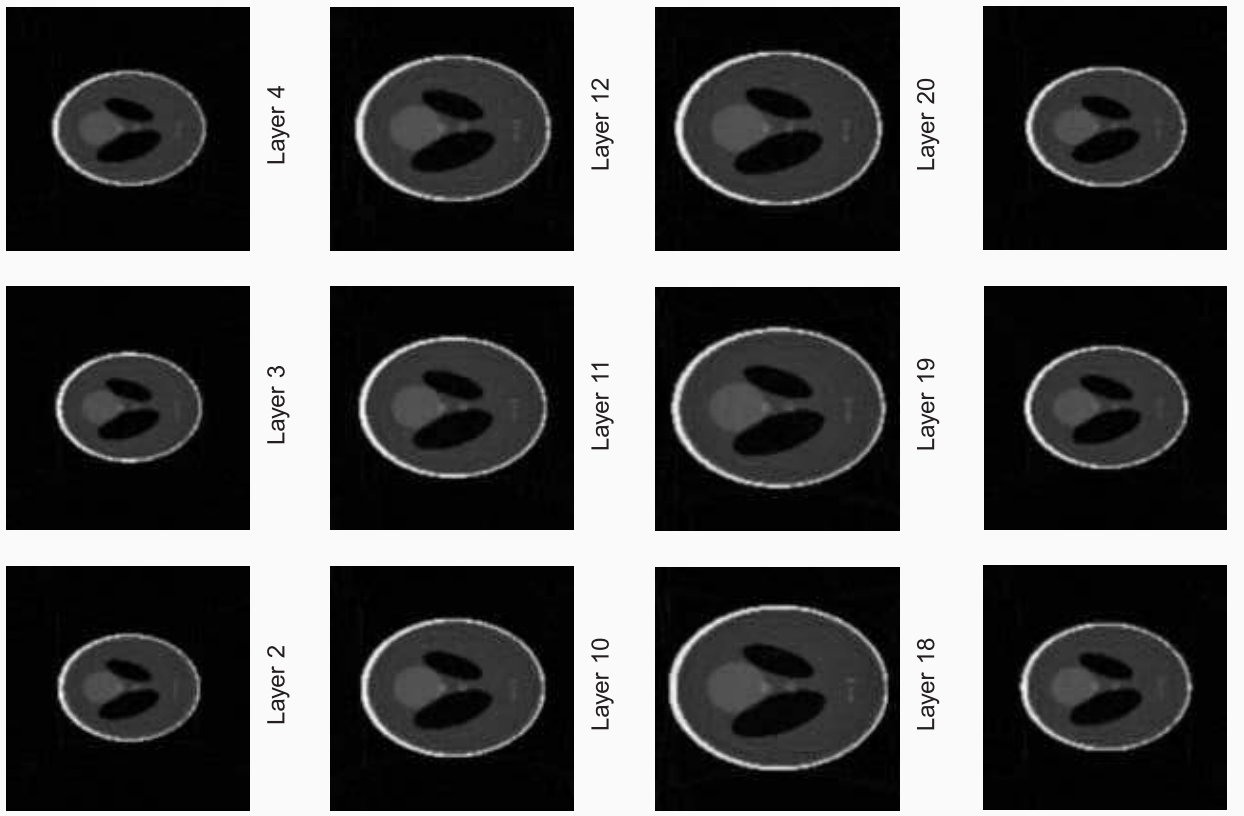

$\frac{2}{2}$
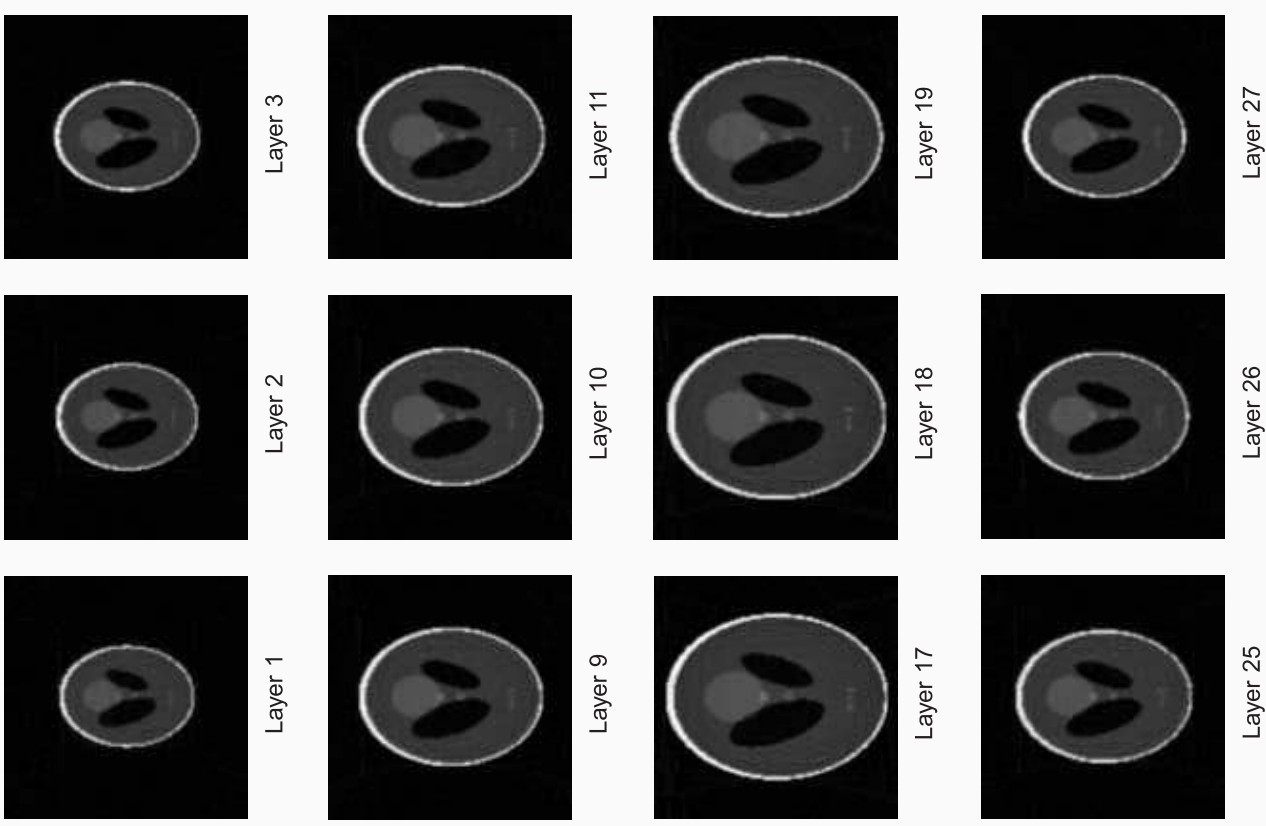


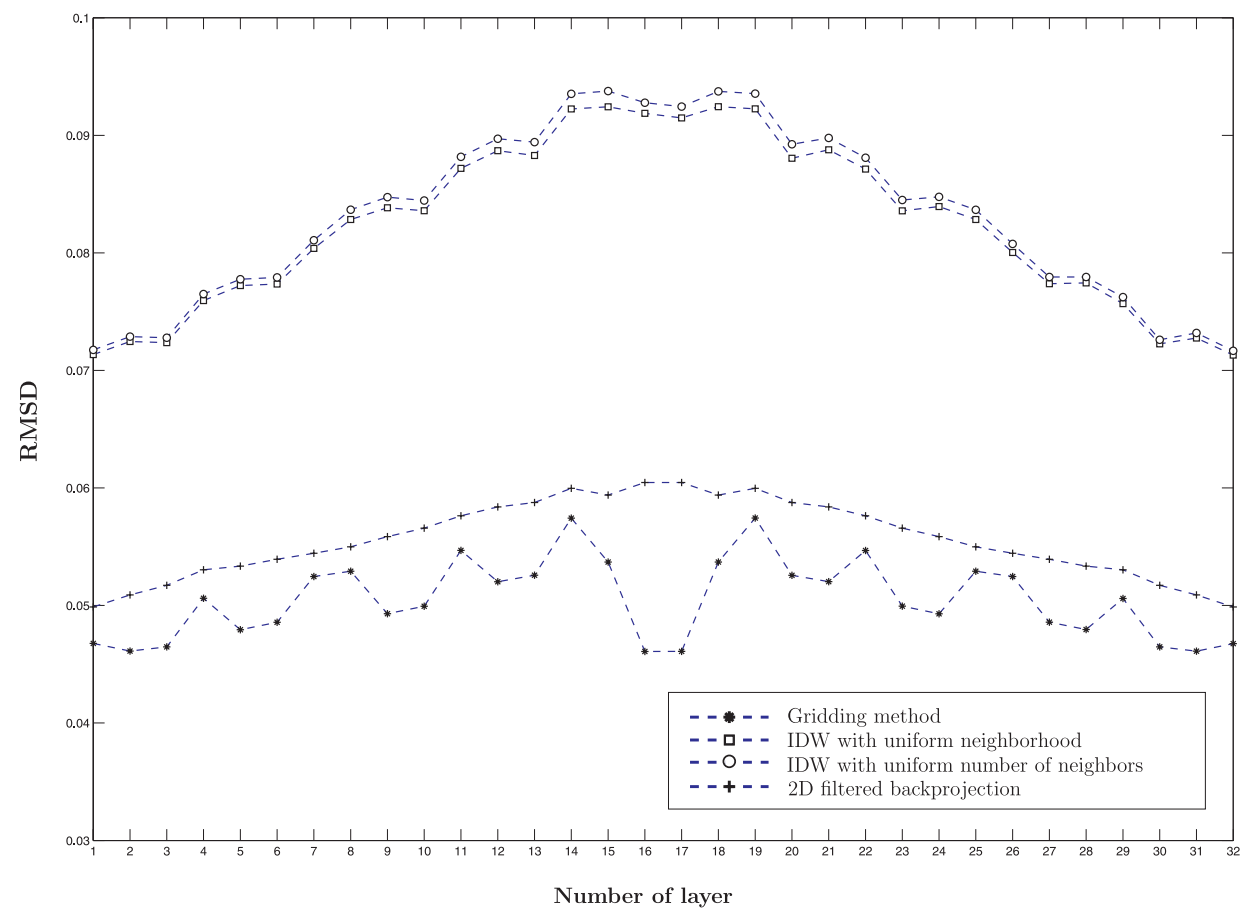

Fig. 12. RMSD values of various reconstruction results.

given dimension, which may appear at first glance not to be very efficient. However, most of the simulation time actually has to be spent to load the pre-calculated weighting factors from the hard disc into the main memory. In this respect, there is no significant time difference between the two reconstruction methods, since no matter how the weighting factors are calculated, they are both pre-calculated and stored on the hard disc as files. Apart from this reading time, the real reconstruction time is in fact much shorter, e.g., a couple of minutes. Nevertheless, further analysis regarding the data arrangement and efficient data input/output is inevitable.

In order to quantify the differences between the diverse reconstructions, the values of the Root-MeanSquare-Deviation (RMSD) for individual reconstruction results can be calculated, with the mathematical definition given by

$$
\operatorname{RMSD}=\sqrt{\frac{1}{M N} \sum_{i=1}^{M} \sum_{j=1}^{N}\left(f_{\text {ori }}[i, j]-f_{\text {rec }}[i, j]\right)^{2}},
$$

where $f_{\text {ori }}[i, j]$ and $f_{\text {rec }}[i, j]$ denote respectively the original and reconstructed pixel values in the $i$-th row and $j$-th column of the same layer with $M$ rows and $N$ columns. In Fig. 12 the calculated RMSD values depending on layer numbers are depicted for various reconstruction results. Besides IDW and the gridding method, the results of the 2D filtered-backprojection serving as a reference are also evaluated. For the IDW method, both the uniform

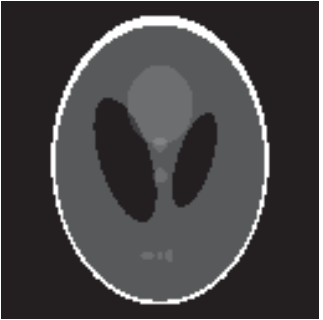

(a)

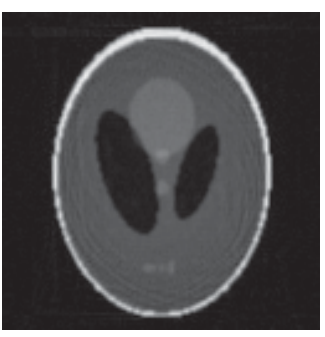

(c)

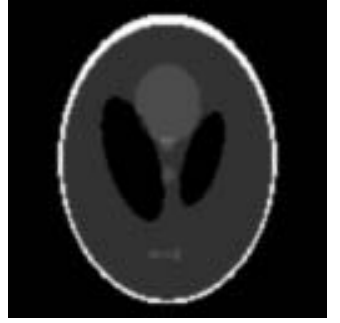

(b)

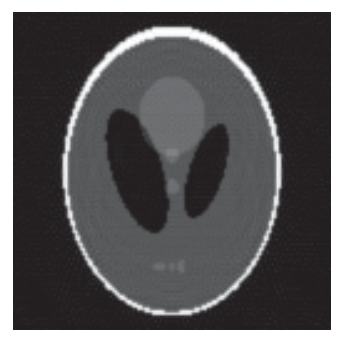

(d)
Fig. 11. Middle layers in the reconstructed 3D phantoms by using the Fourier-based inversion methods and the 2D filtered backprojection: (a) Shepp-Logan phantom, (b) 2D filtered backprojection, (c) inverse distance weighting , (d) gridding method.

neighborhood and the uniform number of neighbors are taken into account. The diagram in Fig. 12 reveals that both of the IDW implementations provide equivalent outcomes, which are, however, worse than that of the FBP 
method, whereas the result achieved by using the gridding method has the best reconstruction quality among all the methods. The reason for the unsatisfying result of the IDW method traces back to the fact that the straightforward interpolation in the frequency domain corresponds to an equivalent low-pass filtering in the space domain, through which artefacts are introduced into the reconstruction results. On the contrary, the gridding method uses convolution instead of direct interpolation and therefore balances the effect of low-pass filtering to some degree. While for the IDW method the weighting factors are exclusively determined by distances between frequency samples and the interpolation location, the gridding method has a much higher degree of freedom due to the versatile choices of convolution functions. In particular, by choosing the parameters of the MKB function properly, a good compromise that both the MKB function in the frequency domain and its inverse transform in the space domain decay relatively rapidly and smoothly can be reached so that the low-pass effect can be compensated more effectively.

\section{Conclusions}

In this work interpolation-based reconstruction methods for 3D-PET were proposed and analyzed. Since merely consecutive $2 \mathrm{D}$ interpolation operations in the $3 \mathrm{D}$ frequency domain are necessary, reconstruction procedures can be sped up significantly. However, simulation studies reveal that classically implemented interpolation does not provide satisfying results, while interpolation performed based upon convolution operations in the frequency domain leads indeed to a better reconstruction quality. Although additional compensating steps in the spatial domain are demanded, the required weighting factors can be calculated in advance and fetched during the convolution procedure so that the thereby caused computational overhead may be even neglected.

\section{Acknowledgments}

This work was supported by the German Research Foundation (DFG) under the grant KU 678/10-2.

\section{References}

Beutel J., Kundel H. L. and Van Metter R. L. (2000). Handbook of Medical Imaging, SPIE Press, Bellingham, Washington.
Bendriem B. and Townsend D. W. (1998). The Theory and Practice of $3 D$ PET, Kluwer Academic Publishers, Dordrecht.

De Berg M., Van Kreveld M., Overmars M. and Schwarzkopf O. (1997). Computational Geometry, Springer-Verlag, Berlin.

Fisher N. I. and Embleton B.J. (1987). Statistical Analysis of Spherical Data, Cambridge University Press, Cambridge.

Fortune S. (1987). A SWEEPLINE algorithm for Voronoi diagrams, Algorithmica 2(2): 153-174.

Jackson J. I., Meyer C. H., Nishimura D.G. and Macovski A. (1991). Selection of a convolution function for Fourier inversion using gridding, IEEE Transactions on Medical Imaging 10(3): 473-478.

Kak A. C. and Slaney M. (1988). Principles of Computerized Tomographic Imaging, New York, IEEE Press.

Li Y., Kummert A., Boschen F. and H. Herzog (2005). Investigation on projection signals in 3D PET systems, Proceedings of the 12th International Conference on Biomedical Engineering, Singapore.

Li Y., Kummert A., Boschen F. and H. Herzog (2005). Spectral properties of projection signals in 3-D tomography, Proceedings of the 16th Triennial World Congress of the International Federation of Automatic Control, Prague, Czech Republic.

Li Y., Kummert A. and Herzog H. (2006). Direct Fourier method in 3D PET using accurately determined frequency sample distribution, Proceedings of the 28th Annual International Conference of the IEEE Engineering in Medicine and Biology Society, New York, USA.

Li Y., Kummert A., Li H. and Herzog H. (2006). Evaluation of the direct Fourier method for 3D-PET in the case of accurately determined projection data, Proceedings of the 11th IASTED International Conference on Signal and Image Processing, Honolulu, USA.

Matej S. and Lewitt R. M. (2001). 3D-FRP: Direct Fourier reconstruction with Fourier reprojection for fully 3-D PET, IEEE Transactions on Medical Imaging 48(4): 1378-1385.

Moon T.K. (1996). The expectation-maximization algorithm, IEEE Signal Processing Magazine 13(6): 47-60.

Schomberg H. and Timmer J. (1995). The gridding method for image reconstruction by Fourier transformation, IEEE Transactions on Medical Imaging 14(3): 596-607.

Thevenaz P., Blu T. and Unser M. (2000). Interpolation revisited, IEEE Transactions on Medical Imaging 19(7): 739-758. 
